\title{
THE EXISTENCE OF FANO SUBPLANES IN GENERALIZED HALL PLANES
}

\author{
Dedicated to the memory of Hanna Neumann
}

A. J. RAHILLY

(Received 1 June 1972)

Communicated by $\mathbf{G}$. Szekeres

\section{Introduction}

One of the best known classes of non-Desarguesian planes is the class of Hall planes (see Hall [2]). In [6] Hanna Neumann showed that the finite Hall planes of odd order possess subplanes of order two (i.e., Fano subplanes) ${ }^{1}$. Kirkpatrick [5] has considered a type of plane which is a generalization of the Hall planes and which he calls generalized Hall planes. In this paper we will give a sufficient condition that a finite generalized Hall plane possesses Fano subplanes. Some examples of odd order planes to which the condition applies shall be exhibited.

We shall assume that the reader is familiar with the elements of projective plane theory and particularly with finite translation planes and Veblen-Wedderburn systems. The reader is referred to Hall [2] and Andre [1] for this. We note that the method of choosing coordinates used in this paper is that due to Hall.

\section{The kernel of a generalized Hall system}

A projective plane $\pi$ is said to be a generalized Hall plane with respect to the line $l_{\infty}$ and the Baer subplane $\pi_{0}$ if $\pi$ is a translation plane with respect to $l_{\infty}, l_{\infty}$ is a line of $\pi_{0}$ and there exists a collineation group $G$ of $\pi$ such that $G$ is sharply transitive on the set of points $l_{\infty} \backslash\left(\pi_{0} \cap l_{\infty}\right)$.

Kirkpatrick [5] shows that a generalized Hall plane of odd order ${ }^{2}$ may be coordinatized by a Veblen-Wedderburn system $F$ with the properties

(1) $F$ is a right vector space of dimension two over a subfield $F_{0}$,

${ }^{1}$ In [6] it is shown that there is a Hall plane of each possible finite odd order possessing Fano subplanes. Hughes (see [3]) has shown that there is a unique Hall plane of each possible finite order.

2 The result is true for even order planes as well. 
(2) There exist two mappings $P, Q$ of $F_{0} \times F_{0}$ onto $F_{0}$ such that $(z \alpha+\beta) z=z P(\alpha, \beta)+Q(\alpha, \beta)$ for all $\alpha, \beta \in F_{0}$ and $z \in F \mid F_{0}$, where

(i) $P$ and $Q$ are additive homomorphisms with $P(0,1)=1$ and $Q(0,1)=0$,

(ii) for any given $\gamma$ and $\delta$ in $F_{0}$, the equation $(P(\alpha, \beta), Q(\alpha, \beta))=(\gamma, \delta)$ has exactly one solution $(\alpha, \beta)$, and

(iii) for any given $\gamma$ and $\delta$ in $F_{0}$, the equation $(P(\alpha, \beta), Q(\alpha, \beta))=(\alpha \gamma, \beta \gamma+\delta)$ has exactly one solution $(\alpha, \beta)$; also, for this solution, $\alpha=0$ if and only if $\delta=0$.

It can be shown that a finite system satisfying (1) and (2) is a Veblen-Wedderburn system which coordinatizes a generalized Hall plane. Such a system is called a generalized Hall system.

The functions $P$ and $Q$ being additive may be written $P(\alpha, \beta)=f(\alpha)+h(\beta)$ and $Q(\alpha, \beta)=g(\alpha)+k(\beta)$ for all $\alpha, \beta \in F_{0}$ where $f, g, h, k$ are additive endomorphisms of $F_{0}$ which we shall the defining functions of $F$. We note that the Hall systems are the special case where $f(\alpha)=r \alpha, g(\alpha)=s \alpha, h(\alpha)=\alpha$ and $k(\alpha)=0$ for all $\alpha \in F_{0}$, where $x^{2}-r x-s$ is an irreducible polynomial over $F_{0}$.

LEMMA 1. The only generalized Hall system which is a field is GF(4).

Proof. Suppose $F$ is a generalized Hall system which is a field. Then $z^{2}=z f(1)+g(1)$ for all $z \in F \backslash F_{0}$. So $F \backslash F_{0}$ contains less than three elements and it readily follows that $F_{0}=G F(2)$ and $F=G F(4)$.

It is easy to verify that $G F(4)$ is, in fact, a generalized Hall system with defining functions $f(\alpha)=\alpha, h(\alpha)=\alpha, g(\alpha)=\alpha$ and $k(\alpha)=0$ for all $\alpha \in G F(2)$.

THEOREM 1. The kernel $\operatorname{Ker}(F)$ of a generalized Hall system $F \neq G F(4)$ is the set

$$
\begin{aligned}
K= & \left\{\lambda \in F_{0} \mid f(\lambda \alpha)=\lambda f(\alpha), g(\lambda \alpha)=\lambda g(\alpha),\right. \\
& \left.h(\lambda \alpha)=\lambda h(\alpha), k(\lambda \alpha)=\lambda k(\alpha) \text { for all } \alpha \in F_{0}\right\} .
\end{aligned}
$$

Proof. If $z \in F \backslash F_{0}$ belongs to $\operatorname{Ker}(F)$ then $z\left(w_{1} w_{2}\right)=\left(z w_{1}\right) w_{2}$ and $z\left(w_{1}+w_{2}\right)=z w_{1}+z w_{2}$ for all $w_{1}, w_{2} \in F$. Suppose $w \in F \mid F_{0}$. There is an automorphism $\theta$ of $F$ such that $z^{\theta}=w$. So $w\left(w_{1} w_{2}\right)=\left(w w_{1}\right) w_{2}$ and $w\left(w_{1}+w_{2}\right)$ $=w w_{1}+w w_{2}$ for all $w_{1}, w_{2} \in F$. Thus $w \in \operatorname{Ker}(F)$ and so $F \backslash F_{0} \subseteq \operatorname{Ker}(F)$. It follows that $F$ is a field and by Lemma $1, F=G F(4)$. Hence, if $F \neq G F(4)$ then $\operatorname{Ker}(F) \subseteq F_{0}$.

Suppose $\lambda \in \operatorname{Ker}(F)$. Then $\lambda(z \alpha+\beta)=\lambda(z \alpha)+\lambda \beta=(\lambda z) \alpha+\lambda \beta$ for all $\alpha$, $\beta \in F_{0}$. So $(z \alpha+\beta) h(\lambda)+k(\lambda)=(z h(\lambda)+k(\lambda)) \alpha+\lambda \beta$ for all $\alpha, \beta \in F_{0}$, whence $(h(\lambda)-\lambda) \beta=k(\lambda)(\alpha-1)$ for all $\alpha, \beta \in F_{0}$ which implies $h(\lambda)=\lambda$ and $k(\lambda)=0$. Thus $\lambda x=x \lambda$ for all $x \in F$. 
Now $\lambda((z \alpha+\beta) z)=(\lambda(z \alpha+\beta)) z$ for all $\alpha, \beta \in F_{0}$ and therefore $[z(f(\alpha)$ $+h(\beta))+g(\alpha)+k(\beta)] \lambda=(z \alpha \lambda+\beta \lambda) z=z(f(\alpha \lambda)+h(\beta \lambda))+g(\alpha \lambda)+k(\beta \lambda)$ for all $\alpha, \beta \in F_{0}$. So $(f(\alpha)+h(\beta)) \lambda=f(\alpha \lambda)+h(\beta \lambda)$ and $(g(\alpha)+k(\beta)) \lambda=g(\alpha \lambda)+k(\beta \lambda)$ for all $\alpha, \beta \in F_{0}$. It readily follows that $f(\alpha) \lambda=f(\alpha \lambda), g(\alpha) \lambda=g(\alpha \lambda), h(\alpha) \lambda=h(\alpha \lambda)$ and $k(\alpha) \lambda=k(\alpha \lambda)$ for all $\alpha \in F_{0}$.

Suppose $\lambda \in K$. Then $\lambda=h(1) \lambda=h(\lambda)$ and $0=k(1) \lambda=k(\lambda)$. So $\lambda x=x \lambda$ for all $x \in F$. It is easy to verify that $\lambda(x+y)=\lambda x+\lambda y$ for all $x, y \in F$. Now $(\lambda(z \alpha+\beta)) z=z(f(\alpha \lambda)+h(\beta \lambda))+g(\alpha \lambda)+k(\beta \lambda)=[z(f(\alpha)+h(\beta))+g(\alpha)$ $+k(\beta)] \lambda=((z \alpha+\beta) z) \lambda=\lambda((z \alpha+\beta) z)$ for all $\alpha, \beta \in F_{0}$ and $z \in F \mid F_{0}$. Clearly $\lambda(x \mu)=(\lambda x) \mu$ for all $x \in F$ and $\mu \in F_{0}$ and so $\lambda \in \operatorname{Ker}(F)$.

COROLlaRY. If $\lambda \in \operatorname{Ker}(F)$, where $F$ is a generalized Hall system, $\lambda x=x \lambda$ for all $x \in F$.

\section{Fano subplanes}

LemMa 2. Let $F$ be a generalized Hall system with defining functions $f, g, h, k$ on the field $F_{0}$. Denote $f(1)$ and $g(1)$ by $r$ and $s$ respectively. If $r$ and $s \in \operatorname{Ker}(F), r+2 s-1=0$ and $l=r+s-1$ then

(i) $\left(l^{-1}(u+s)\right)(u+1)-u=0$ and

(ii) $\left(l^{-1}(u+s)\right) u-u-l^{-1}(u+s)=0$

for all $u \in F \backslash F_{0}$.

Proof. Firstly, $l \neq 0$ since $r+s-1=r+2 s-1$ implies $s=0$ and so $z^{2}=z r$ for all $z \in F \backslash F_{0}$ which implies $z=0$ or $r$ for all $z \in F \backslash F_{0}$, a contradiction.

We note also that $l \in \operatorname{Ker}(F)$ since $r$ and $s \in \operatorname{Ker}(F)$.

(i)

$$
\begin{aligned}
\left(l^{-1}(u+s)\right)(u+1)-u & =l^{-1}((u+s)(u+1))-u \\
& =l^{-1}((u+1+s-1)(u+1))-u \\
& =l^{-1}\left((u+1)^{2}+(s-1)(u+1)\right)-u \\
& =l^{-1}(l u+r+2 s-1)-u \text { (using the cor- } \\
& =0, \quad \text { ollary to Theorem } 1)
\end{aligned}
$$

(ii) $\left(l^{-1}(u+s)\right) u-u-l^{-1}(u+s)$

$$
\begin{aligned}
& =l^{-1}((u+s) u)-u-l^{-1}(u+s) \\
& =l^{-1}((u+s) u-(u+s))-u \\
& =l^{-1}(l u)-u \text { (using the corollary to Theorem } 1 \text { again) } \\
& =0 .
\end{aligned}
$$


THEOREM 2. Let $\pi$ be a generalized Hall plane coordinatized by the generalized Hall system $F$ with defining functions $f, g, h, k$ on the field $F_{0}$. Suppose $f(1)=r$ and $g(1)=s$ belong to $\operatorname{Ker}(F)$ and $r+2 s-1=0$. Then the quadrangle $Y=(\infty), 0=(0,0), I=(1,1), \quad R=\left(l^{-1}(u+s), 0\right)$, where $l=r+s-1$, generates a Fano subplane of $\pi$ for all $u$ in $F \backslash F_{0}$.

Proof. Clearly $Y I \cap O R=(1,0)$ and $O I \cap Y R=\left(l^{-1}(u+s), l^{-1}(u+s)\right)$. The line $I R$ has equation $y=x(u+1)-u$ since the coordinates of $R$ satisfy it (by Lemma 2 (i)) and those of I clearly do. Thus $I R \cap O Y=(0,-u)$.

The line $(0,-u)(1,0)$ has equation $y=x u-u$. Now $\left(l^{-1}(u+s), l^{-1}(u+s)\right)$ lies on this line (by Lemma 2(ii)). So the quadrangle $Y, 0, I, R$ generates a Fano subplane of $\pi$.

\section{Examples}

In this section we shall give some examples of odd order generalized Hall planes to which Theorem 2 applies.

1. Hall planes. If $F_{0}$ is a finite field of odd order $r$ and $s$ may be chosen such that $r+2 s-1=0$ and $x^{2}-r x-s$ is irreducible over $F_{0}$ (see [6], p. 39). The kernel of the Hall system with defining functions $f(\alpha)=r \alpha, g(\alpha)=s \alpha, h(\alpha)$ $=\alpha$ and $k(\alpha)=0$ is $F_{0}$. So we see by Theorem 2 that the planes over such systems possess Fano subplanes. These planes, in fact, constitute the entire class of odd order Hall planes. The Fano subplanes just given are those discovered by Hanna Neumann [6].

2. Let $F_{0}=G F\left(p^{2 n}\right)$ where $p$ is an odd prime.

(a) $f(\alpha)=r^{\theta-1} \alpha, g(\alpha)=s \alpha^{\theta} h(\alpha)=\alpha^{\theta-1}$ and $k(\alpha)=0$ and

(b) $f(\alpha)=r^{\theta-1} \alpha^{\theta-1}, g(\alpha)=s \alpha^{\theta-1}, h(\alpha)=\alpha^{\theta-1}$ and $k(\alpha)=0$, where $\theta$ is an automorphism of $F_{0}$, are sets of defining functions giving rise to two classes of generalized Hall systems provided that $x^{\theta} x-r x-s$ is irreducible over $F_{0}$. These systems appear in Johnson [4] in another form. The planes they coordinatize are the planes derived from some well known semifield planes using Ostrom's derivation process. ${ }^{3}$

Suppose in (a) and (b) we choose $\theta$ as the involutory automorphism $\phi$ of $F_{0}$, $s \in G F\left(p^{n}\right)=F^{\prime}$ such that $x^{2}-(1-2 s) x-s$ is irreducible over $F^{\prime}([6]$ p. 39) and $r=1-2 s$. Then $p(x)=x^{\phi} x-r x-s$ is irreducible over $F_{0}$. If $r \neq 0, p(x)$ is irreducible over $F_{0}$ since $x^{\phi} x \in F^{\prime}$ for all $x \in F_{0} \mid F^{\prime}$. If $r=0, p(x)$ is reducible over $F_{0}$. However the restriction $r \neq 0$ is not a serious one.

The generalized Hall systems defined by this choice of $r, s$ and $\theta$ have $f(1)$ $=(1-2 s)^{\phi}=1-2 s$ and $g(1)=s$ and so $f(1)+2 g(1)-1=0$. Also it is easy to show by applying Theorem 1 that $\operatorname{Ker}(F)=F^{\prime}$. From Theorem 2 we see that

\footnotetext{
${ }^{3}$ Ostrom [7] is a good reference for the process of derivation of planes.
} 
the planes coordinatized by these generalized Hall systems possess Fano subplanes.

\section{Acknowledg nents}

The work appearing in this paper was done when the author was a research student on a Commonwealth Post Graduate Award. The author wishes to thank his supervisor Dr. P. B. Kirkpatrick for his guidance and encouragement.

\section{References}

[1] J. André, 'Über nicht-Desarguessche Ebenen mit transitiver Translationsgruppe', Math. $Z$. 60 (1954), 156-186.

[2] M. Hall, Jr. The Theory of Groups (Macmillan, New York, 1959).

[3] D. R. Hughes, 'Collineation groups of non-desarguesian planes. I. The Hall Veblen-Wedderburn systems', Amer. J. Math. 81 (1959), 921-938.

[4] N. L. Johnson, 'Translation planes constructed from semifield planes', Pacific J. Math. (1971), 701-712.

[5] P. B. Kirkpatrick, 'Generalization of Hall planes of odd order', Bull. Austral. Math. Soc. 4 (1971), 205-209.

[6] Hanna Neumann, 'On some finite non-desarguesian planes', Arch. Math. 6 (1955), 36-40.

[7] T. G. Ostrom, 'Semi-translation planes', Trans. Amer. Math. Soc. 111 (1964), 1-18.

University of Sydney

Sydney, New South Wales

Australia 